\title{
On the holographic phase transitions at finite topological charge
}

\author{
Tran Huu Phat ${ }^{1, \mathrm{a}}$, Toan T. Nguyen ${ }^{2}$ (B) \\ ${ }^{1}$ Vietnam Atomic Energy Commission, 59 Ly Thuong Kiet, Hanoi, Vietnam \\ ${ }^{2}$ Key Laboratory for Multiscale Simulation of Complex Systems, University of Science, Vietnam National University, Hanoi, \\ 334 Nguyen Trai Street, Thanh Xuan District, Hanoi, Vietnam
}

Received: 4 July 2020 / Accepted: 14 April 2021 / Published online: 18 May 2021

(C) The Author(s) 2021

\begin{abstract}
Exploring the significant impacts of topological charge on the holographic phase transitions and conductivity we start from an Einstein-Maxwell system coupled with a charged scalar field in Anti-de Sitter spacetime. In our set up, the corresponding black hole (BH) is chosen to be the topological AdS one where the pressure is identified with the cosmological constant (Kubiznak and Mann in JHEP 7:33, 2012), then the AdS BH undergoes the phase transition from small to large BHs, which is totally similar to the transition from gas to liquid in the van der Waals theory. Our numerical computation shows that the process of condensation is favored at finite topological charge, in particular, the phase transition from small to large BHs in the bulk generates a mechanism for changing the order of phase transition in the boundary: the second order phase transitions occur at pressures higher than the critical pressure of the phase transition from small to large BHs while they become first order at lower pressures. This property is confirmed with the aid of holographic free energy. Finally, the frequency dependent conductivity exhibits an energy gap when the phase transition is second order and when the phase transition becomes first order this gap is either reduced or totally lost.
\end{abstract}

\section{Introduction}

It is known that the AdS/CFT duality [1] formulated by Maldacena and developed independently by Witten [2] and Gubser, Klebanov and Polyakov [3] in the form of the GKPW relation has opened up a new direction of connecting gravity with other branches of physics. At present the GKPW relation turns out to be a powerful formalism for this purpose. In this respect, the study of holographic phase transitions has been developed strongly and gained great successes associated with superconductors and related topics [4-17]. However, to our understanding, up to now maybe Ref. [16] was

a e-mail: thphat@live.com (corresponding author) solely the one which suggested that the Einstein-Maxwell system coupled with a charged scalar field in the topological AdS BH could yield superconductivity. Therefore inspired by [16], we will investigate systematically in this paper the holographic phase transitions at finite topological charges. Our main aim is to look for the new effects which could emerge when there occurs the phase transition from small to large BHs in the bulk.

Then let us start from the model of a Abelian Higgs field and a Maxwell field in the four-dimensional spacetime Einstein gravity. The bulk action reads

$$
\begin{aligned}
S= & \frac{1}{16 \pi G_{N}} \int d^{4} x \sqrt{-g}\left(R-\frac{6}{L^{2}}-\frac{1}{4} F_{\mu \nu}^{2}\right. \\
& \left.-\left|\partial_{\mu}-i q A_{\mu} \psi\right|^{2}-m|\psi|^{2}\right),
\end{aligned}
$$

where $G_{N}$ is the Newton constant. In the uncondensed phase, the solutions to Eq. (1) are the Reissner-Nordstrom black hole $(\mathrm{BH})$

$d s^{2}=-f(r) d t^{2}+\frac{d r^{2}}{f(r)}+r^{2} d \Omega_{2, k}^{2}$,

where

$f(r)=k-\frac{M}{r}+\frac{Q^{2}}{r^{2}}+\frac{r^{2}}{L^{2}}$,

and

$\phi(r)=Q\left(\frac{1}{r}-\frac{1}{r_{0}}\right)$,

$\psi(r)=0$,

here the horizon radius, $r_{0}$, is the largest solution of the equation:

$f\left(r_{0}\right)=k-\frac{M}{r_{0}}+\frac{Q^{2}}{r_{0}^{2}}+\frac{r_{0}^{2}}{L^{2}}=0$. 
In Eq. (1), $d \Omega_{2, k}^{2}$ is the metric of a two-sphere of radius $1 / \sqrt{k}$ for $k>0$. Note that the parameters $M$ and $Q$ are different from the mass and the charge of $\mathrm{BH}$ by corresponding factors. The Hawking temperature $T$ and entropy of $\mathrm{BH}$ are respectively given by

$$
\begin{aligned}
& T=\frac{f^{\prime}\left(r_{0}\right)}{4 \pi}, \\
& S=\pi r_{0}^{2} .
\end{aligned}
$$

It is very interesting to mention that adopting the relation between pressure $\mathrm{P}$ and the cosmological constant of $\mathrm{BH}$

$P=-\frac{\Lambda}{8 \pi}=\frac{3}{8 \pi L^{2}}$,

one discovered the total analogy between the small-large BH phase transition and the liquid-gas phase transition of the van der Waals theory for $k>0[11,12]$. In this set up $M$ becomes the enthalpy of the system and $k$ is interpreted as the measure of a new charge, the topological charge $[13,14]$. Then the extended first law [15] of BH reads

$d M=T d S+\omega d \varepsilon+V d P+\phi d Q$,

where $T$ and $S$ are given in Eq. (4a), $\varepsilon=4 \pi k$ is the topological charge and its conjugate potential $\omega=r_{0} / 8 \pi$, the pressure $P$ conjugates to the volume $V=\frac{4}{3} \pi r_{0}^{3}$, the charge $Q$ conjugates to the potential $\phi=Q / r_{0}$.

In term of $\varepsilon$ from Eqs. (3), (4b) and (5), it is easily derived the isobaric specific heat

$C_{P Q \varepsilon}=T\left(\frac{\partial S}{\partial T}\right)_{P Q \varepsilon}=\frac{2 \pi r_{0}^{2}\left(32 \pi^{2} \operatorname{Pr}_{0}^{4}+\varepsilon r_{0}^{2}-4 \pi Q^{2}\right)}{32 \pi^{2} \operatorname{Pr}_{0}^{4}-\varepsilon r_{0}^{2}+12 \pi Q^{2}}$,

which yields the critical values for pressure, temperature and horizon event radius

$P_{c}=\frac{\varepsilon^{2}}{1536 \pi^{3} Q^{2}}, T_{c}=\frac{\sqrt{6} \varepsilon^{3 / 2}}{96 \pi^{3 / 2} Q}, r_{c}=\frac{2 \sqrt{6 \pi} Q}{\sqrt{\varepsilon}}$,

where, for simplicity we set $G_{N}=Q=1$ from now on.

Equation (6) shows that at fixed $\varepsilon$ the transition from liquid to gaz takes place at $P \leq P_{c}$ and $T \geq T_{c}$ and the bigger values of $\varepsilon$ lead to the smaller values of $P$.

The present paper is organized as follows. Section 2 deals with the holographic phase transitions at finite topological charges and the free energy, respectively. Section 3 is devoted to the calculations of frequency dependent conductivity in different phase transitions. The conclusion and outlook are given in Sect. 4.

\section{Holographic phase transitions}

\section{A. Basic set up}

At first let us set up the frame work for the whole study of holographic phase transitions. We begin with the following ansatz

$A_{\mu}=(\phi(r), 0,0,0), \psi=\psi(r)$,

and at the same time we choose

$m^{2}=-\frac{2}{L^{2}}$,

which is above the Breitenlohner-Freedman bound [18].

Inserting Eq. (7)) into Eq. (1) we derive the equations of motion for matter fields

$\phi^{\prime \prime}+\frac{2}{r}+\phi^{\prime}-\frac{2 \psi^{2}}{f} \phi=0$,

$\psi^{\prime \prime}+\left(\frac{2}{r}+\frac{f^{\prime}}{f}\right) \psi^{\prime}+\left(\frac{\phi^{2}}{f^{2}}+\frac{2}{L^{2} f}\right) \psi=0$,

where the prime denotes derivative with respect to $r$.

For the fields to be regular at horizon we impose the condition

$\phi\left(r_{0}\right)=0$.

Inserting Eq. (11) into Eq. (10), and expand near $r \rightarrow r_{0}$, we arrive at the condition at horizon for scalar field

$\frac{\psi^{\prime}\left(r_{0}\right)}{\psi\left(r_{0}\right)}=-\frac{1}{2 \pi L^{2} T}$.

At the AdS boundary, the large $r$ behaviors of $\phi$ and $\psi$ take the form

$\psi(r)=\frac{\psi_{1}}{r}+\frac{\psi_{2}}{r^{2}}+\cdots$

$\phi(r)=\mu-\frac{\rho}{r}+\cdots$,

where $\mu$ and $\rho$ are chemical potential and the corresponding density associated with the expectation value of charge density, $\rho=\left\langle J^{0}\right\rangle$, with a source term in the boundary action of the form

$S_{b d y} \rightarrow S_{b d y}+\mu \int d^{4} x J^{0}(x)$.

Due to the holographic duality there are two possibilities for identifying the sources and condensates of the dual field theory

$-\psi_{1}$ is the source which vanishes at infinity

$\psi_{1}=0$,

and $\psi_{2}$ is condensate $\psi_{2} \sim\left\langle O_{2}\right\rangle$. 
$-\psi_{2}$ is the source which vanishes at infinity

$$
\psi_{2}=0
$$

and $\psi_{1}$ is condensate $\psi_{1} \sim\left\langle O_{1}\right\rangle$.

\section{B. Free energy}

In order to analyse the order of phase transitions we have to calculate the holographic free energy. This quantity is holographically evaluated by calculating the corresponding on-shell value of the Abelian-Higgs sector of the Euclidean action. Plugging the ansatz, Eq. (7), into the action, Eq. (1), we arrive at

$$
\begin{aligned}
& S_{M} \\
& =\int d^{4} x\left[\frac{\phi^{\prime 2}(z)}{2}-f(z) \psi^{\prime 2}(z)+\frac{\phi^{2}(z) \psi^{2}(z)}{z^{4} f(z)}+\frac{2 \psi^{2}(z)}{z^{4}}\right] .
\end{aligned}
$$

Here we have made a change of variable, $z=1 / r$, to better formulate the system of equations of motion for numerical evaluations and to simplify various expressions.

Applying the boundary condition, Eq. (11), and the Eqs. $(9,10)$, we obtain the on-shell value of the Euclidean action

$$
\begin{aligned}
S_{O S}= & \int \frac{1}{k}\left(\left.\frac{\phi^{\prime}(z) \psi(z)}{z^{2}}\right|_{z=0}-\left.\frac{f(z) \psi^{\prime}(z) \psi(z)}{z}\right|_{z=0}\right) d^{3} x \\
& -\int \frac{1}{k}\left(\int_{0}^{z_{0}} d z \frac{\phi^{2}(z) \psi(z)}{z^{4} f(z)}\right) d^{3} x .
\end{aligned}
$$

Substituting the asymptotic behaviors of $\phi$ and $\psi$ into the above action we get

$$
\begin{aligned}
S_{O S}= & \int \frac{1}{k}\left(\frac{\mu \rho}{2}+\frac{3 \psi_{1} \psi_{2}}{L^{2}}+\left(\frac{\psi_{1}^{2}}{z L^{2}}\right)_{z=0}\right) d^{3} x \\
& -\int \frac{1}{k}\left(\int_{0}^{z_{0}} d z \frac{\phi^{2}(z) \psi^{2}(z)}{z^{4} f(z)}\right) d^{3} x .
\end{aligned}
$$

The divergence term in the foregoing expression will be removed by adding the counter term [8]

$$
S_{c}=-\frac{1}{k L^{2}} \int d^{3} x\left(\sqrt{-h} \psi^{2}(z)\right)_{z=0}
$$

where $h$ is the determinant of the induced metric on the AdS boundary. With the aid of the asymptotic behavior of $\psi$ it is easily found that

$$
S_{c}=-\frac{1}{k L^{2}} \int d^{3} x\left[2 \psi_{1} \psi_{2}+\left(\frac{\psi_{1}^{2}}{z}\right)_{z=0}\right] \text {. }
$$

The renormalised free energy of the boundary field theory is obtained

$$
\begin{aligned}
\Omega_{1} & =-T\left(S_{O S}+S_{C}\right) \\
& =V_{2}\left[-\frac{\mu \rho}{2}-\frac{O_{1} O_{2}}{L^{2}}+\int_{0}^{z_{0}} d z \frac{\psi^{2}(z) \phi^{2}(z)}{z^{4} f(z)}\right],
\end{aligned}
$$

which corresponds to the $O_{1}$ quantization, $V_{2}$ is the volume of two-sphere with radius $1 / \sqrt{k}$.

Analogously, the renormalised free energy in $\mathrm{O}_{2}$ quantization reads

$\Omega_{2}=V_{2}\left[-\frac{\mu \rho}{2}+\frac{O_{1} O_{2}}{L^{2}}+\int_{0}^{z_{0}} d z \frac{\psi^{2}(z) \phi^{2}(z)}{z^{4} f(z)}\right]$,

which is the Legendre transform of $\Omega_{1}$. From Eqs. (18) and (19), it is clear that

$\frac{1}{V_{2}} \frac{\partial \Omega_{1}}{\partial O_{1}}=-O_{2}, \quad \frac{1}{V_{2}} \frac{\partial \Omega_{2}}{\partial O_{2}}=O_{1}$

implying that the local extrema of $\Omega_{1}\left(\Omega_{2}\right)$ locates at vanishing $O_{2}\left(O_{1}\right)$. This is exactly what we assumed that only one of $\psi_{1}$ and $\psi_{2}$ is non-vanishing for physical solutions.

The free energy corresponding to non-condensed state reads

$\frac{\Omega_{0}}{V_{2}}=\frac{\mu^{2} z_{0}}{2}$.

From Eq. (20), we get the free energy difference

$$
\frac{\Delta \Omega_{i}}{V_{2}}=\frac{\Omega_{i}}{V_{2}}-\frac{\mu^{2} z_{0}}{2} \quad(i=1,2) .
$$

This is our expected result.

\section{Numerical results}

In this subsection we focus on the impacts of the topological charge in the holographic phase transitions. To this end, let us proceed to the numerical calculation which will be implemented in the following cases:

$$
\text { 1. } \varepsilon=4 \pi, L=1
$$

The scalar condensation is plotted in Fig. 1, where the onset of second order phase transitions occur at $T_{c}=0.113 \mu$ and $0.050 \mu$, respectively.

After fitting the curves in Fig. 1 near $T \rightarrow T_{c}$, we obtain approximately the expressions

$$
\frac{\left\langle O_{1}\right\rangle}{T_{c}}=\frac{\sqrt{2} \psi_{1}}{T_{c}} \approx 11.318\left(1-\frac{T}{T_{c}}\right)^{1 / 2}
$$


Fig. 1 The condensation of $\left\langle O_{1}\right\rangle$ (a) and $\left.\rangle O_{2}\right\rangle$ (b)

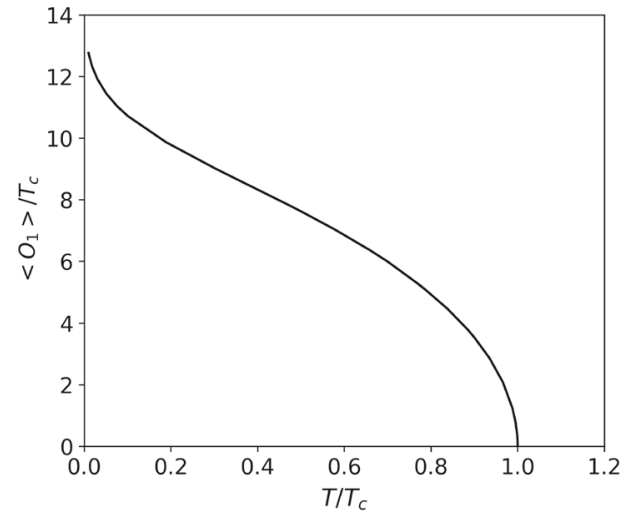

(a)

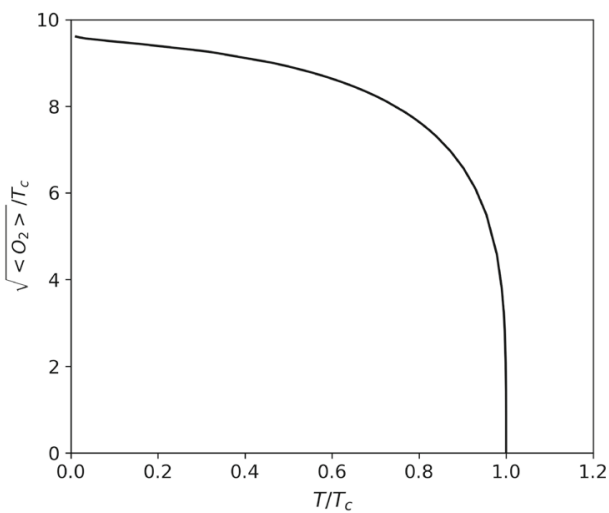

(b) as $T \rightarrow T_{c}=0.113 \mu$ and

$\frac{\sqrt{\left\langle O_{2}\right\rangle}}{T_{c}}=\frac{\sqrt{\sqrt{2} \psi_{2}}}{T_{c}} \approx 32.966\left(1-\frac{T}{T_{c}}\right)^{1 / 2}$.

as $T \rightarrow T_{c}=0.050 \mu$.

Figure 1 and the foregoing expressions characterise one of the typical properties of superconductors in mean-field approximation. It is worth to note that the phase transition takes place at $L=1$, corresponding to pressure $P_{L=1}=$ $\left(\frac{3}{8 \pi L^{2}}\right)_{L=1}$ which is bigger than the critical pressure $P_{c}=$ $\left(\frac{3}{8 \pi L^{2}}\right)_{L=6}$ given in Eq. (6).

Now let the pressure decrease to lower values which corresponds to bigger values of $L$. In Fig. $2 \mathrm{a}, \mathrm{b}$ are shown the phase diagrams for $L=1,4,6$, and 9. It is clear that at critical pressure $P_{c}=\left(\frac{3}{8 \pi L^{2}}\right)_{L=6}$, the first order phase transition begins to appear.

In order to confirm exactly the exhibition of first order phase transitions let us calculate numerically the free energy difference, Eq. (21), between the condensed and uncondensed phases for $O_{1}$ and $O_{2}$, respectively. They are plotted in Fig. 3a, b which indicate that at $L=6,9$ the free energy difference is not analytical at corresponding critical temperatures.

2. $\varepsilon=16 \pi$ and $L=0.5,1.5,2$

Next we consider the case when the topological charge takes bigger value. The phase diagrams plotted In Fig. 4 tell that at $L \geq 1.5$ the first order phase transitions emerge.

Figure 4 demonstrate again that the first order phase transitions always emerge at critical pressure $P_{c}=\left(\frac{3}{8 \pi L^{2}}\right)_{L=1,5}$ and moreover when the topological charge gets bigger values the first order phase transitions manifest at higher pressures. The existence of the first order phase transitions in Fig. 4 are confirmed again in Fig. 5 the free energy is not analytical at corresponding temperatures.

\section{Conductivity}

Let us finally proceed to the conductivity of superconductor in the dual CFT as a function of frequency. For this purpose, we must solve the equation for the fluctuations of the vector potential $A_{x}(r, t)$ in the bulk. Let this potential take the form

$A_{x}(r, t)=A_{x}(r) \exp (-i \omega t)$,

from which we get the equation of $A_{x}(r)$ in our set up

$A^{\prime \prime}{ }_{x}(r)+\frac{f^{\prime}}{f} A^{\prime}{ }_{x}(r)+\left(\frac{\omega^{2}}{f^{2}}-\frac{2 \psi^{2}}{f}\right) A_{x}(r)=0$,

which is rewritten in new variable $z=1 / r$ as

$$
\begin{aligned}
& A^{\prime \prime}{ }_{x}(z)+\left(\frac{2}{z}+\frac{f^{\prime}}{f}\right) A^{\prime}{ }_{x}(z) \\
& \quad+\left(\frac{\omega^{2}}{z^{4} f^{2}}-\frac{2 \psi^{2}}{z^{4} f}\right) A_{x}(z)=0,
\end{aligned}
$$

where $\psi$ was solved in Sect. 2. In order to determine the solution to Eqs. $(22,23)$ we need two boundary conditions. The first one is given by the on going condition at horizon

$A_{x}(r) \sim\left(r-r_{0}\right)^{-i \omega / 4 \pi T}+\cdots$

and the second boundary condition is set at large $r$. To do this, $A_{x}$ is expanded in term of $z$ near $z \rightarrow 0$. This gives

$A_{x}(z)=a_{x}+z b_{x}+\cdots$

Therefore the second boundary condition is

$A_{x}(0)=a_{x}$.

The AdS/CFT duality dictionary tells that $b_{x}$ determines the boundary current $j_{x}=b_{x}$. Then the Ohm law gives us the conductivity

$\sigma(\omega)=\frac{j_{x}}{E_{x}}=-\frac{i b_{x}}{\omega a_{x}}$.

Equation (27) requires us to solve the differential equation $(22,23)$ based on two boundary conditions (24) and (25). The 
Fig. 2 For $L<6$ the phase transitions are second order while for $L \geq 6$ the first order phase transitions begin to exhibit for $O_{1}$ (up) and $O_{2}$ (down)

Fig. 3 The free energy difference is not analytical at corresponding critical temperatures. a (b) corresponds to $O_{1}\left(O_{2}\right)$

Fig. 4 For $L<1.5$ phase transition is second order. It turns out to be first order at $L=1.5$. See $O_{1}$ (up) and $O_{2}$ (down)

Fig. 5 The free energy difference calculated for $O_{1}$ quantization (up) and $\mathrm{O}_{2}$ quantization (down) it is not analytical at corresponding critical temperatures

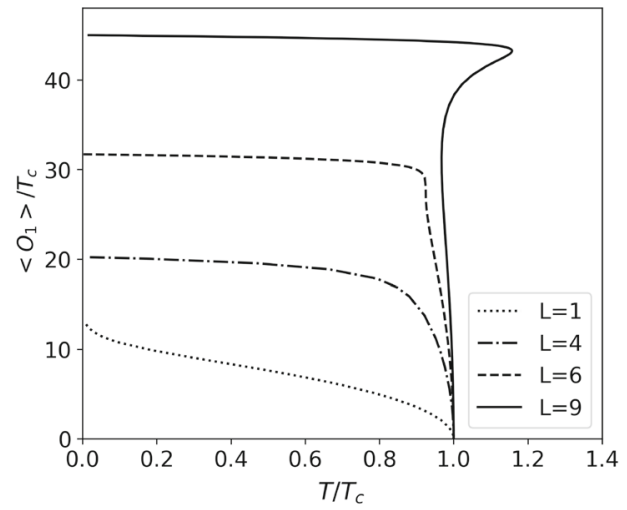

(a)

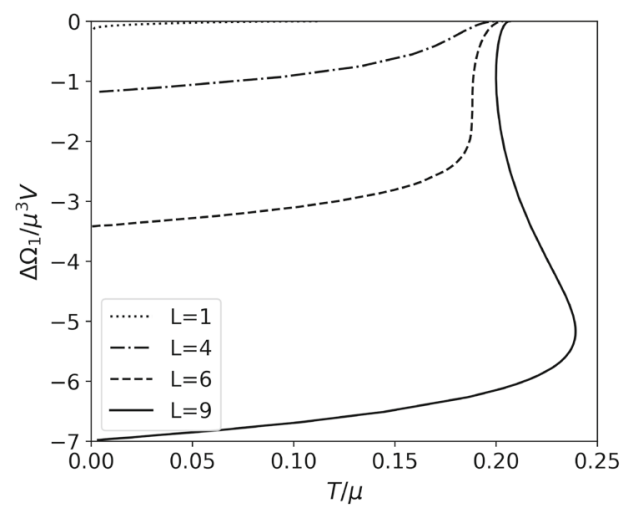

(a)

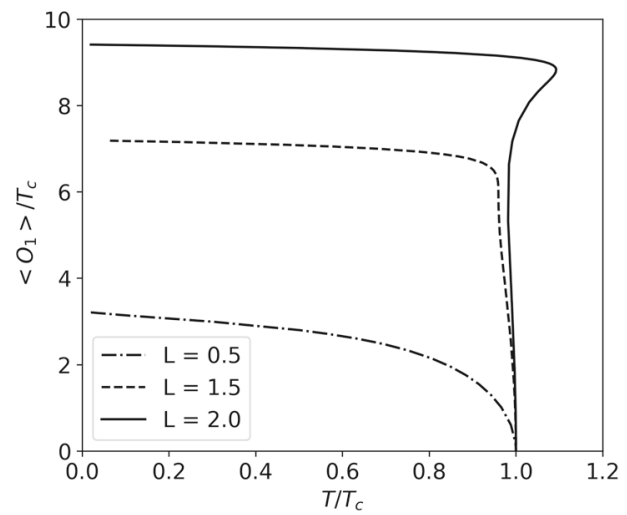

(a)

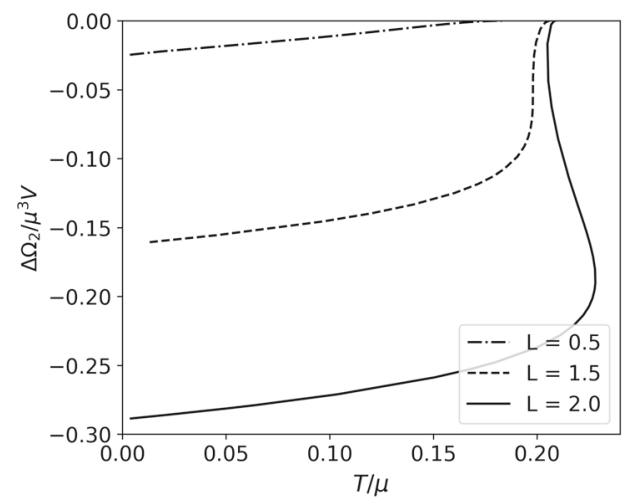

(a)

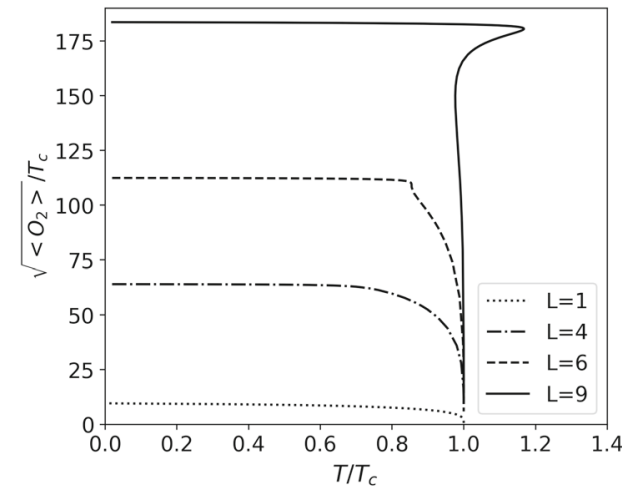

(b)

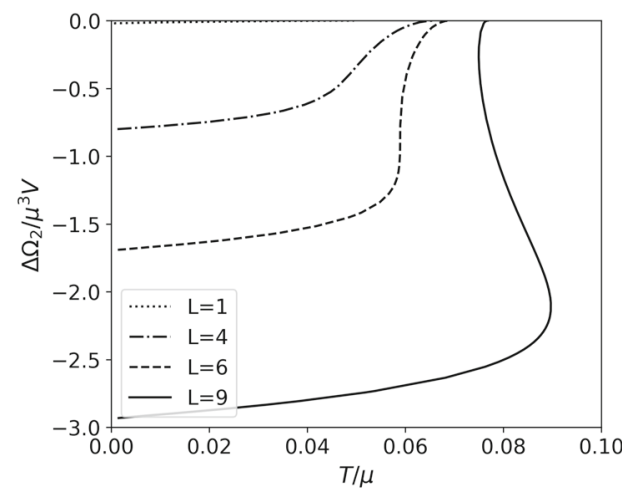

(b)

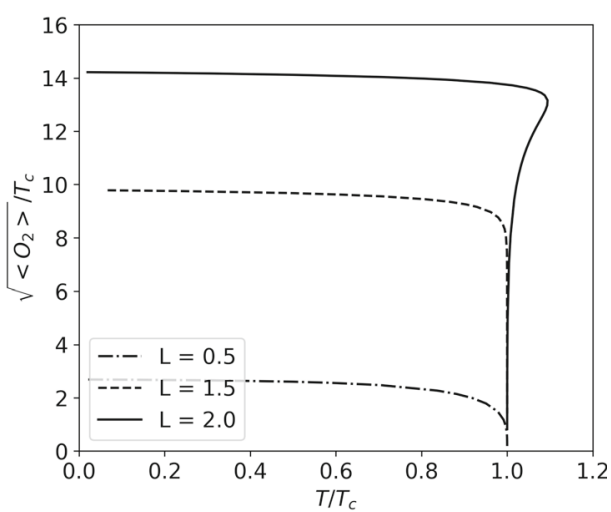

(b)

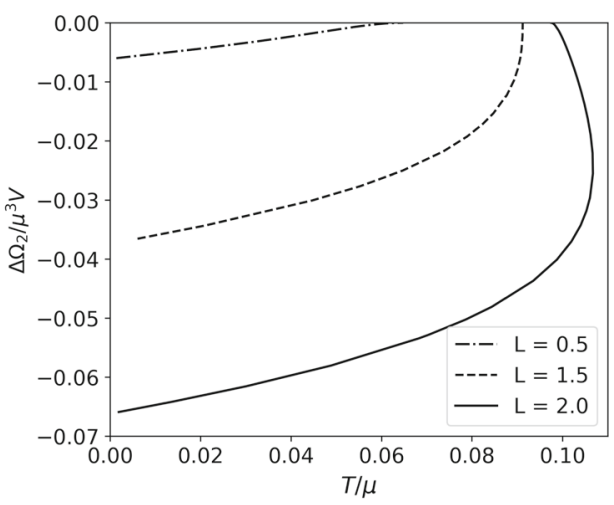

(b) 


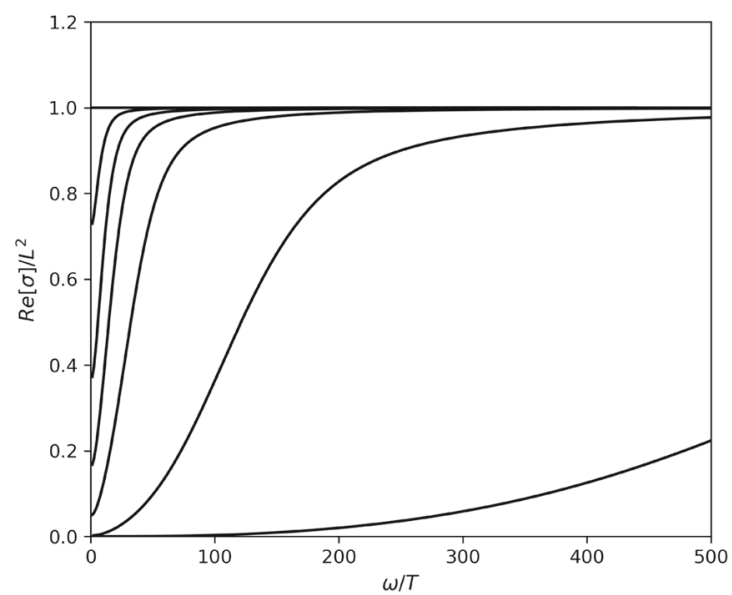

(a)

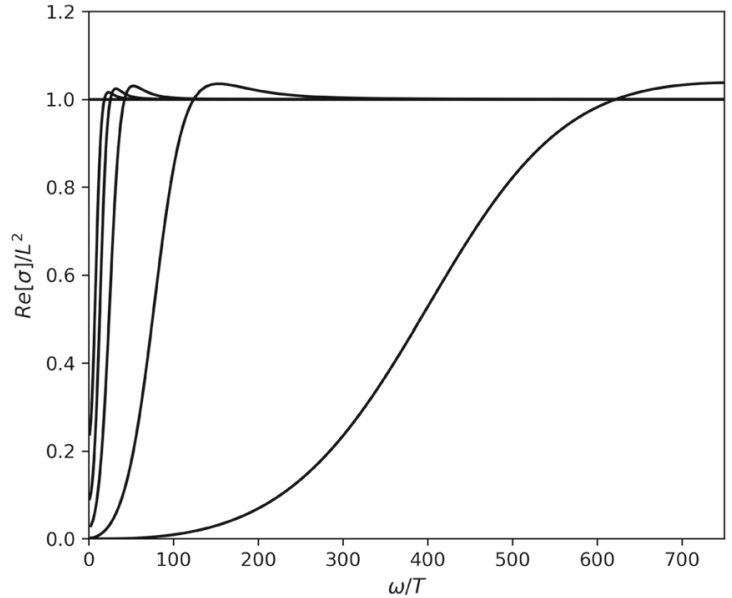

(b)

Fig. 6 The evolution of $\operatorname{Re} \sigma(\omega)$ versus $\omega / T$ at $\varepsilon=4 \pi, Q=L=1$ for $O_{1}$ (a) and $O_{2}$ (b). Each graph, from left to right, is plotted for $T / T_{c}=$ $0.9,0.7,0.5,0.3,0.1$ and 0.02 respectively

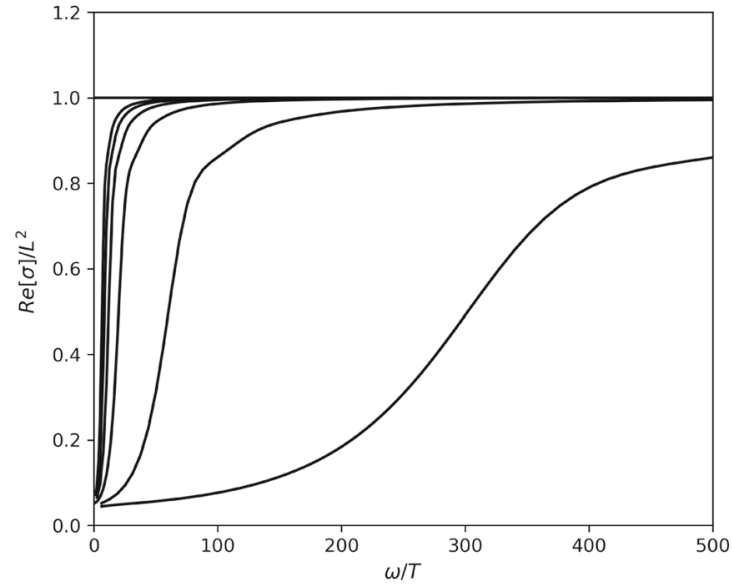

(a)

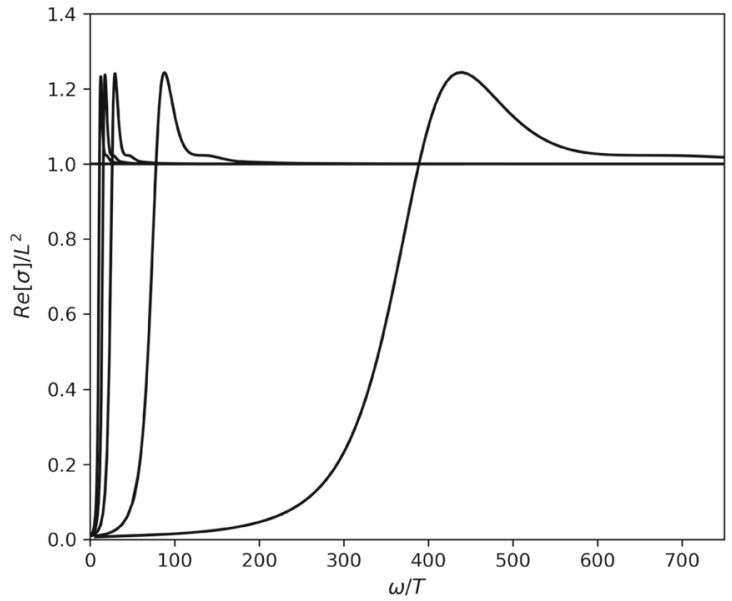

(b)

Fig. 7 The evolution of $\operatorname{Re} \sigma(\omega)$ versus $\omega / T$ at $\varepsilon=4 \pi, Q=1, L=9$ for $O_{1}$ (a) and $O_{2}$ (b). Each graph, from left to right, is plotted for the cases $T / T_{c}=0.7,0.5,0.3,0.1$ and 0.02 respectively

numerical computation provides the graphs of the frequency dependent conductivity corresponding respectively to the phase diagrams given in subsection $C$. They are presented in accordance with different items in subsection $C$ :

1. $\varepsilon=4 \pi, Q=1, L=1$, and 9

We plot the real part of frequency dependent conductivity $\operatorname{Re} \sigma(\omega)$ derived from the numerical calculations of Eqs. (22, 23) at $Q=k=L=1$ in Fig. 6a, b which exhibit a gap determined by condensate and, furthermore $\operatorname{Re} \sigma(\omega)$ contains a delta function which is recognized from the imaginary part $\operatorname{Im} \sigma(\omega)$ having a pole at $\omega=0$ (it is not shows up here). Therefore the real part and imaginary part of $\omega=0$ are related by the Kramers-Kronig relations. At $L=9$ we have correspondingly Fig. 7a, b which tell that the foregoing gap disappears. This means that the superconductivity is totally lost.
2. $\varepsilon=16 \pi, Q=1, L=0.5$, and 2

Corresponding to $L=0.5$ we depict the graphs in Fig. 8a, $\mathrm{b}$ which prove clearly that the gap is really reduced even when the phase transition is second order, this implies that a mixture of normal and conductive states emerges. At $L=2$ the gap totally disappears as seen in Fig. 9a, b, the superconductivity is totally lost. Thus, the conductivity of superconductor is greatly affected by topological charge. These features are similar to those of some strongly coupled superconductors [19-21].

\section{Conclusion and discussion}

Before summarizing this paper it is worth to emphasize again that the existence of the topological charge leads to the phase 


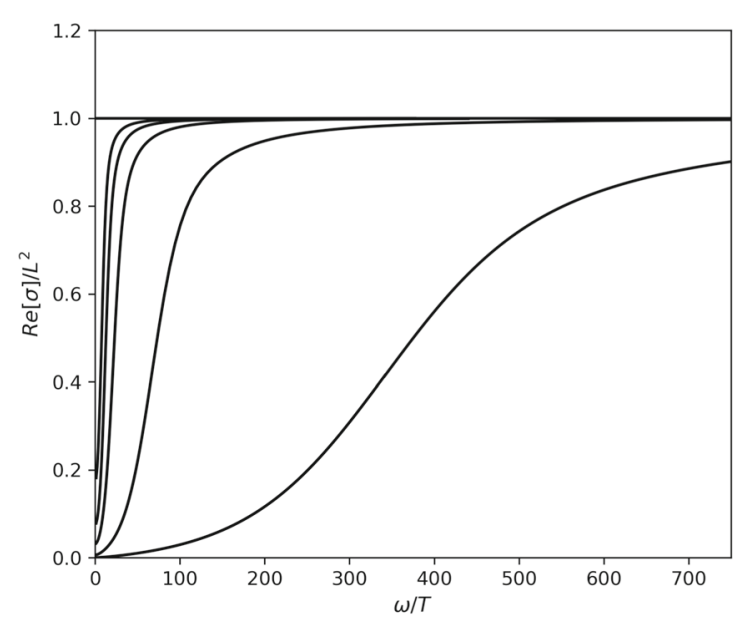

(a)

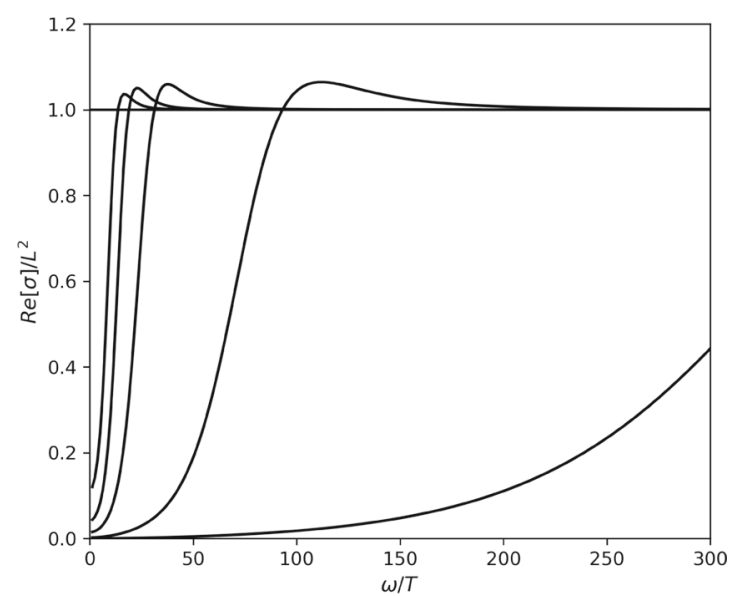

(b)

Fig. 8 The evolutions of $\operatorname{Re} \sigma(\omega)$ versus $\omega / T$ at $\varepsilon=16 \pi, L=0.5$ for $O_{1}$ (a) and $O_{2}$ (b). Each graph, from left to right, is plotted for the cases $T / T_{c}=0.7,0.5,0.3,0.1$ and 0.02 respectively

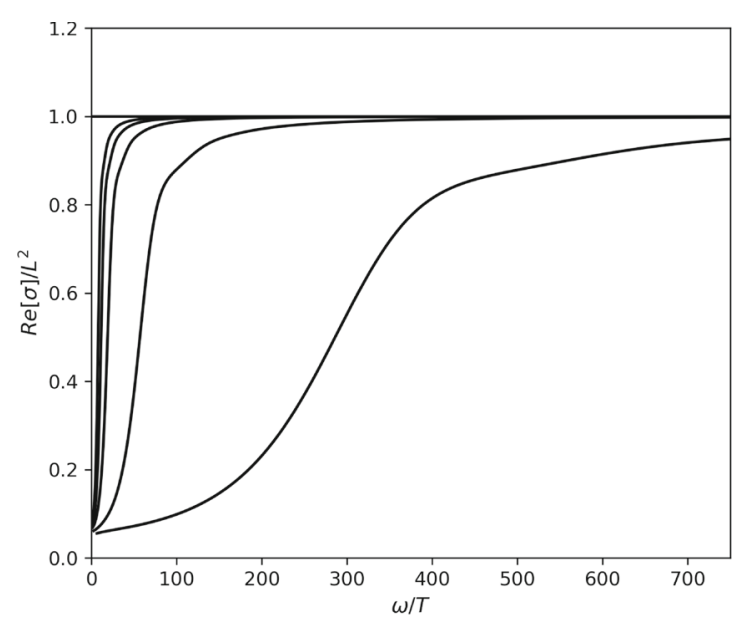

(a)

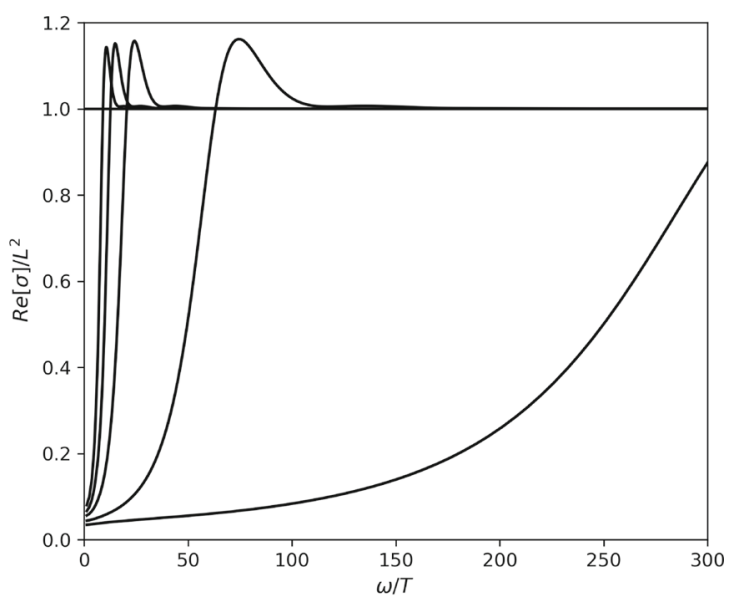

(b)

Fig. 9 The evolution of $\operatorname{Re} \sigma(\omega)$ versus $\omega / T$ at $\varepsilon=16 \pi, L=2$ for $O_{1}$ (a) and $O_{2}$ (b). Each graph, from left to right, is plotted for the cases $T / T_{c}=0.7,0.5,0.3,0.1$ and 0.02 respectively

transitions from small to large $\mathrm{BHs}$ which totally similar the phase transition from liquid to gas in the van der Waals theory. Within this framework we studied the significant impact of the topological charge on the holographic phase transitions and what we obtained can be brefly written as follows.

1. The phase transition from small to large $\mathrm{BHs}$ in the bulk created a mechanism for changing the holographic phase transitions from second order to first order in the boudary. In other words, the holographic phase transitions in the boundary are closely associated with the phase transition from liquid to gaz in the van der Waals theory. This effect was confirmed by the free energy difference.

2. When the phase transitions are second order the frequency dependent conductivity $\sigma(\omega)$ exhibits an energy gap and, moreover, its real and imaginary parts fulfill the Kramers - Kronig relation while the phase transition becomes first order the gap is either reduced or totally lost. Our set up provides a new types of holographic superconductivity in which the topological charge plays the crucial role. The foregoing results exactly express the novelty of our paper, they do not exist for BHs without topological charge. Therefore they express the significant impacts of topological charge.

It is very interesting to remark that there exists a formal analogy between our present paper and Ref. [9] although our starting points are different, namely,

The holographic phase transitions studied in our paper is associated with the transition from small to large BHs in the bulk while in Ref. [9] the holographic phase transitions is associated with the Hawking-Page transition in the bulk because the AdS-soliton decays into AdS BH via HawkingPage transition. 
We start from the charged AdS BH in four dimensional spacetime with finite topological charge while Ref. [9] started from the AdS-soliton in five dimensional spacetime without topological charge, as a consequence, the holographic transition is solely second order.

The analogous effect was also presented in other papers, for example, Refs. [6,7] with different mechanisms. In Ref. [7] the charged scalar field is forced to condense by another neutral scalar field and in Ref. [6] the nonlinear interaction of charged scalar field was employed $F=\sum_{2}^{4} c_{i}|\psi|^{i}$.

One found that first order phase transitions occurred for $c_{4} \geq 1$ and, moreover, the gap becomes narrower as $c_{4}$ increases from 0 to 1 .

Reference [22] dealing with the unbalanced Stackelberg holographic superconductors the authors showed that depending on the values of the Stackelberg model's parameters the phase transitions also change from second to first order and, at the same time, the conductivity gaps are affected strongly.

Last but not least it is clear that the results found above allows us to have a better understanding on the physical meaning of topological charge. In this regard, we need to explore more and more the the impacts of this charge on various physical processes. The backreaction of matter fields will be the subject of our next research.

Acknowledgements This paper was supported by the Vietnam National Foundation for Science and Technology Development under the Grant No. 103.01-2017.300. We thank P.H.Lien, N. T. Anh, L. V. Hoa and H. V. Quyet for useful discussions.

Data Availability Statement This manuscript has no associated data or the data will not be deposited. [Authors' comment: This is a theoretical study and no experimental data has been listed.]

Open Access This article is licensed under a Creative Commons Attribution 4.0 International License, which permits use, sharing, adaptation, distribution and reproduction in any medium or format, as long as you give appropriate credit to the original author(s) and the source, provide a link to the Creative Commons licence, and indicate if changes were made. The images or other third party material in this article are included in the article's Creative Commons licence, unless indicated otherwise in a credit line to the material. If material is not included in the article's Creative Commons licence and your intended use is not permitted by statutory regulation or exceeds the permitted use, you will need to obtain permission directly from the copyright holder. To view a copy of this licence, visit http://creativecomm ons.org/licenses/by/4.0/.

Funded by SCOAP ${ }^{3}$.

\section{References}

1. J.M. Maldacena, Adv. Theor. Math. Phys. 2, 231 (1998)

2. E. Witten, Adv. Theor. Math. Phys. 2, 253 (1998)

3. S.S. Gubser, I.R. Klebanov, A.M. Polyakov, Phys. Lett. B 428, 105 (1998)

4. S.A. Hartnoll, C.P. Herzog, G.T. Horowitz, Phys. Rev. Lett. 101, 031601 (2008)

5. G.T. Horowitz, M.M. Roberts, Phys. Rev. 78, 126008 (2008)

6. P. Basu, J. Bhattacharya, S. K. Das. arXiv: 1906.02452

7. S. Franco, A. M. Garcia - Garcia, D. Rodriguez - Gomez, Phys. Rev. D 81, 041901 (2010)

8. C.P. Herzog, P.K. Kovtun, D.T. Son, Phys. Rev. D 79, 066002 (2009)

9. T. Nishioka, S. Ryu, T. Takayanagi, JHEP 03, 131 (2010)

10. J. Zaanen, Y.W. Sun, Y. Liu, K. Schalm, Holographic Duality in Condensed Matter Physics (Cambridge University Press, Cambridge, 2015)

11. M. Ammon, J. Erdmenger, Gauge/Gravity Duality: Foundations and Applications (Cambridge University Press, Cambridge, 2015)

12. D. Kubiznak, R. Mann, JHEP 07, 033 (2012)

13. D. Kubiznak, R. Mann, M. Teo, Class. Quant. Grav. 34, 063001 (2017)

14. Y. Tian, X.N. Wu, H.B. Zhang, JHEP 10, 170 (2014)

15. Y. Tian, The last (lost) charge of black hole. arXiv:1804.00249

16. S. Q. Lan, Adv. High Energy Phys. 2018, Article ID 350287

17. S.S. Gubser, Phys. Rev. D 78, 065034 (2008)

18. P. Breitenlohner, D.Z. Freedman, Ann. Phys. (NY) 144, 249 (1982)

19. D.S. Fisher et al., Phys. Rev. B 43, 130 (1991)

20. M.L. Horbach et al., Phys. Rev. B 46, 130 (1992)

21. K. Holcze et al., Phys. Rev. Lett. 67, 152 (2001)

22. A. J. Hafshejan, S. A. Hossein Mansoori. arXiv:1808.02628 